\title{
Contribution of surface polysaccharides to the resistance of Campylobacter jejuni to antimicrobial phenolic compounds
}

\author{
Euna Oh and Byeonghwa Jeon \\ The Journal of Antibiotics (2015) 68, 591-593; doi:10.1038/ja.2015.26; published online 11 March 2015
}

Campylobacter spp., a particular Campylobacter jejuni, is a leading bacterial cause of food-borne gastroenteritis worldwide. ${ }^{1}$ In addition, the increasing resistance of $C$. jejuni to clinically important antibiotics for the treatment of human campylobacteriosis, such as fluoroquinolones and macrolides, has become a serious public health concern worldwide. ${ }^{2}$ Several molecular mechanisms for antibiotic resistance that have been reported in $C$. jejuni include the alteration of cellular targets for antibiotics, ${ }^{3}$ removal of antibiotics by efflux pumps, ${ }^{4}$ enzymes that hydrolyze or modify antibiotics ${ }^{5}$ and limitation of entry of antibiotics into the cell. ${ }^{6}$

Phenolic compounds are a group of secondary metabolites naturally present in a wide range of plants. They have been extensively investigated owing to their antioxidant properties and beneficial health effects, such as anticancer and antimicrobial activities. ${ }^{7}$ Antibacterial effects of phenolic compounds have been reported in several different food-borne pathogens, including Campylobacter, and the antimicrobial activities of phenolic compounds vary depending on the structure of phenolic compound and the bacterial strain. ${ }^{8,9}$ Although a recent study showed that the multidrug efflux pump CmeABC has an important role in the resistance of $C$. jejuni to phenolic antimicrobials, ${ }^{10}$ the molecular mechanisms for resistance to phenolic antimicrobials have not yet been defined well in Campylobacter. In this study, we compared the anti-Campylobacter activities of two major classes of phenolic compounds, such as flavonoids and phenolic acids, and demonstrated the role of surface polysaccharides in the resistance of C. jejuni to phenolic antimicrobials.

Five $C$. jejuni strains were used in this study, including two genomesequenced C. jejuni strains (NCTC 11168 and 81-176), ${ }^{11,12}$ two human clinical isolates (HCJ3599 and HCJ2082), and a poultry isolate (PCJ497). C. jejuni strains were routinely grown with Mueller-Hinton (MH) media at $42{ }^{\circ} \mathrm{C}$ under microaerobic conditions $\left(5 \% \mathrm{O}_{2}, 10 \%\right.$ $\mathrm{CO}_{2}, 85 \% \mathrm{~N}_{2}$ ). Occasionally, culture media were supplemented with kanamycin $\left(50 \mu \mathrm{g} \mathrm{ml}^{-1}\right)$ and tetracycline $\left(5 \mu \mathrm{g} \mathrm{ml}^{-1}\right)$. As surface polysaccharides are an important permeability barrier against antibiotics, we investigated their role in resistance to phenolic antimicrobials. In this study, we used previously reported kpsS and waaF mutants. The $k p s S$ gene encodes a $\beta$-Kdo-transferase (Kdo: 3-deoxy-Dmanno-octulosonic acid) that has a key role in the CPS synthesis. ${ }^{13}$ The waaF gene encodes a heptosyltransferase, the inactivation of which results in the truncation of the inner core moiety of LOS in $C$. jejuni; ${ }^{14}$ therefore, the $k p s S$ mutant (kpsS::aphA3) and the waaF mutant (waaF::tetO) produce truncated CPS and LOS, respectively. ${ }^{6}$ The waaF mutants of 81-176, PCJ497, HCJ3599 and HCJ2082 were constructed by natural transformation with the genomic DNA of the waaF mutant of NCTC 11168 as described previously. ${ }^{6}$ Briefly, the recipient $C$. jejuni strains were grown overnight on $\mathrm{MH}$ agar and resuspended in $\mathrm{MH}$ broth to an $\mathrm{OD}$ at $600 \mathrm{~nm}$ of 0.1 . After shaking incubation at 200 r.p.m. for $3 \mathrm{~h}$, the genomic DNA of the waaF mutant was added to the culture. The $C$. jejuni strains were further grown for $6 \mathrm{~h}$ and plated on $\mathrm{MH}$ agar containing $5 \mu \mathrm{g} \mathrm{ml}^{-1}$ of tetracycline to select the waaF mutants. The minimum inhibitory concentrations (MICs) of phenolic antimicrobials were determined with a microtiter broth dilution method. ${ }^{4}$ All phenolic compounds were purchased from Sigma-Aldrich (St Louis, MI, USA).

Flavonoids, such as morin, quercetin, hesperidin and taxifolin, exhibited antimicrobial effects on C. jejuni NCTC 11168 (Table 1). The anti-Campylobacter activities of synthetic phenolic compounds, including butylated hydroxytoluene, lauryl gallate and propyl gallate, were also examined. The synthetic phenolic compounds are considered to be generally recognized as safe and often used as antioxidant food additives to prevent lipid oxidation. ${ }^{15}$ Interestingly, these synthetic phenolic compounds demonstrated a high anti-Campylobacter activity in comparison with natural phenolic compounds (Table 1). We also investigated the role of surface polysaccharides in $C$. jejunis resistance against antimicrobial phenolic compounds by using CPS and LOS mutants. C. jejuni possesses CPS and LOS on the bacterial surface. Unlike other Gram-negative bacteria that usually harbor lipopolysaccharides (LPS) consisting of lipid A, core and O-antigen structures, the LOS of $C$. jejuni does not contain an O-antigen polysaccharide chain. ${ }^{16}$ Mutations of CPS and LOS increased the susceptibility of C. jejuni NCTC 11168 to phenolic compounds (Table 1). An LOS mutation (that is, the waaF mutant) reduced 
Table 1 Susceptibility of surface polysaccharide mutants of $C$. jejuni NCTC 11168 to phenolic antimicrobials

\begin{tabular}{|c|c|c|c|c|c|c|}
\hline \multirow[b]{2}{*}{ Class } & \multirow[b]{2}{*}{ Subclass } & \multirow[b]{2}{*}{ Compound } & \multicolumn{4}{|c|}{ C. jejuni NCTC $11168^{a}$} \\
\hline & & & $W T$ & $\Delta k p s S$ & $\Delta w a a F$ & waaF comp $p^{\mathrm{b}}$ \\
\hline \multirow[t]{8}{*}{ Phenolic acid } & Hydroxycinnamic acid & p-Coumaric acid & 1024 & $512(2)$ & $128(8)$ & 1024 \\
\hline & & Caffeic acid & 1024 & $512(2)$ & $256(4)$ & 1024 \\
\hline & & Sinapic acid & 512 & $256(2)$ & $64(8)$ & 512 \\
\hline & Hydroxybenzoic acid & Vanillic acid & 1024 & $512(2)$ & $512(2)$ & 1024 \\
\hline & & Salicylic acid & 512 & 512 & $128(4)$ & 512 \\
\hline & & Gallic acid & 512 & 512 & $128(4)$ & 512 \\
\hline & & Lauryl gallate & 256 & $128(2)$ & $16(16)$ & 256 \\
\hline & & Propyl gallate & 128 & $64(2)$ & $16(8)$ & $256(1 / 2)$ \\
\hline \multirow[t]{7}{*}{ Flavonoid } & Flavan-3-ol & Epigallocatechin gallate & 512 & $256(2)$ & $64(8)$ & 512 \\
\hline & Flavonol & Morin & 256 & 256 & $32(8)$ & $512(1 / 2)$ \\
\hline & & Quercetin & 256 & $128(2)$ & $64(4)$ & $512(1 / 2)$ \\
\hline & Flavone & Chrysin & 1024 & $512(2)$ & $512(2)$ & 1024 \\
\hline & Flavanone & Naringenin & 512 & 512 & $256(2)$ & 512 \\
\hline & & Hesperidin & 256 & $128(2)$ & $32(8)$ & 256 \\
\hline & Flavanolol & Taxifolin & 256 & $128(2)$ & $64(4)$ & 256 \\
\hline Other phenolic & & Butylated hydroxytoluene & 256 & $128(2)$ & $16(16)$ & 256 \\
\hline
\end{tabular}

Abbreviation: WT, wild type.

The table shows MIC ( $\mu \mathrm{g} \mathrm{ml}^{-1}$ ) and fold changes in parenthesis.

aThe results are representative of three independent experiments that produced similar results.

${ }^{\mathrm{b}} \mathrm{A}$ waaF complementation strain.

Table 2 Increased susceptibility to phenolic antimicrobials by an LOS mutation in multiple $C$. jejuni strains

\begin{tabular}{|c|c|c|c|c|c|c|c|c|c|c|}
\hline \multirow[b]{3}{*}{ Class } & \multirow[b]{3}{*}{ Subclass } & \multirow[b]{3}{*}{ Compound } & \multicolumn{8}{|c|}{ C. jejuni strains ${ }^{\mathrm{a}}$} \\
\hline & & & \multicolumn{2}{|c|}{$81-176$} & \multicolumn{2}{|c|}{ PCJ497 } & \multicolumn{2}{|c|}{ HCJ3599 } & \multicolumn{2}{|c|}{ HCJ2082 } \\
\hline & & & $W T$ & $\Delta w a a F$ & $W T$ & $\Delta$ waaF & $W T$ & $\Delta$ waaF & $W T$ & $\Delta$ waaF \\
\hline \multirow[t]{8}{*}{ Phenolic acid } & \multirow[t]{3}{*}{ Hydroxycinnamic acid } & p-Coumaric acid & 512 & $256(2)$ & 512 & $256(2)$ & 512 & $256(2)$ & 512 & $256(2)$ \\
\hline & & Caffeic acid & 1024 & $128(8)$ & 512 & $256(2)$ & 512 & $256(2)$ & 512 & $256(2)$ \\
\hline & & Sinapic acid & 512 & $128(4)$ & 512 & $256(2)$ & 256 & $128(2)$ & 256 & $128(2)$ \\
\hline & \multirow[t]{5}{*}{ Hydroxybenzoic acid } & Vanillic acid & 512 & $256(2)$ & 512 & $128(4)$ & 512 & $256(2)$ & 512 & $256(2)$ \\
\hline & & Salicylic acid & 512 & $256(2)$ & 512 & $256(2)$ & 1024 & $256(4)$ & 1024 & $256(4)$ \\
\hline & & Gallic acid & 1024 & $256(4)$ & 1024 & $256(4)$ & 512 & $128(4)$ & 512 & $256(2)$ \\
\hline & & Lauryl gallate & 256 & $128(2)$ & 512 & $128(4)$ & 512 & $64(8)$ & 512 & $64(8)$ \\
\hline & & Propyl gallate & 256 & $64(4)$ & 256 & $64(4)$ & 512 & $64(8)$ & 512 & $128(4)$ \\
\hline \multirow[t]{7}{*}{ Flavonoid } & Flavan-3-ol & Epigallocatechin gallate & 512 & $128(4)$ & 512 & $256(2)$ & 256 & $128(2)$ & 512 & $128(4)$ \\
\hline & \multirow[t]{2}{*}{ Flavonol } & Morin & 512 & $128(4)$ & 256 & 256 & 512 & $128(4)$ & 512 & $256(2)$ \\
\hline & & Quercetin & 256 & $128(2)$ & 256 & $128(2)$ & 128 & $32(4)$ & 128 & $32(4)$ \\
\hline & Flavone & Chrysin & 256 & $128(2)$ & 512 & $256(2)$ & 512 & $256(2)$ & 256 & $128(2)$ \\
\hline & \multirow[t]{2}{*}{ Flavanone } & Naringenin & 256 & $64(4)$ & 256 & $64(4)$ & 128 & $32(4)$ & 256 & $64(4)$ \\
\hline & & Hesperidin & 512 & $64(8)$ & 512 & $128(4)$ & 256 & $128(2)$ & 256 & 256 \\
\hline & \multirow[t]{2}{*}{ Flavanolol } & Taxifolin & 512 & $128(4)$ & 256 & 256 & 256 & $128(2)$ & 256 & $64(4)$ \\
\hline Other phenolic & & Butylated hydroxytoluene & 256 & $128(2)$ & 256 & $128(2)$ & 128 & 64 (2) & 128 & $32(4)$ \\
\hline
\end{tabular}

Abbreviation: WT, wild type.

The table shows MIC $\left(\mu \mathrm{g} \mathrm{ml}^{-1}\right)$ and fold changes in parenthesis.

aThe results are representative of three independent experiments that produced similar results.

MIC levels in all the tested phenolic antimicrobials, whereas a CPS mutation (that is, the $k p s S$ mutant) presented only minor MIC changes in some phenolic compounds (Table 1). In particular, the LOS mutation resulted in 16-fold MIC reductions in butylated hydroxytoluene and lauryl gallate and 8 -fold MIC reductions in p-coumaric acid, sinapic acid, propyl gallate, epigallocatechin gallate, morin and hesperidin (Table 1). Also, the MIC changes in the LOS mutant were successfully restored to the wild-type level by complementation (Table 1), suggesting that the increased susceptibility to phenolic antimicrobials by the LOS mutation is specifically related to the function of LOS. The results show that LOS is the major permeability barrier conferring resistance to phenolic antimicrobials. As the hypervariability in surface polysaccharides has been reported in C. jejuni, ${ }^{12}$ we also determined the effect of an LOS mutation on the susceptibility to phenolic antimicrobials in different $C$. jejuni strains. Anti-Campylobacter activities of phenolic compounds were confirmed 
in several C. jejuni strains (Table 2). Particularly, quercetin, naringenin and butylated hydroxytoluene consistently exhibited active antiCampylobacter activities in the strains (Table 2), and the LOS mutation in all the strains reduced the MIC levels of phenolic compounds (Table 2).

The outer membrane and the surface polysaccharides are an important permeability barrier to toxic compounds in Gram-negative bacteria. ${ }^{17}$ The LPS structures that are embedded in the outer leaflet of the outer membrane often contribute to bacterial resistance to hydrophobic antibiotics. ${ }^{18}$ In Campylobacter, the LOS confers resistance to not only hydrophobic drugs (for example, erythromycin $)^{6}$ but also bile salts, affecting C. jejuni colonization of chicken intestines. ${ }^{19}$ In this study, we successfully demonstrated that LOS also significantly contributed to the resistance of $C$. jejuni to phenolic antimicrobials. Presumably, the effect of LOS in the resistance would result from direct inference with the attachment of hydrophobic molecules to hydrophilic LOS structures on the bacterial surface. In addition, direct or indirect interaction with membrane proteins, such as drug efflux pumps, would affect the resistance role of LOS in C. jejuni. It has been reported that the multidrug efflux pump AcrB and LPS synergistically contribute to the intrinsic resistance of Salmonella. ${ }^{20}$ As the multidrug efflux pump CmeABC has an important role in C. jejuni resistance to phenolic compounds, ${ }^{10}$ we cannot exclude the possibility that LOS would interplay with $\mathrm{CmeABC}$ in $C$. jejuni resistance to phenolic compounds. Given the increasing number of newly reported phenolic antimicrobials from various sources, the findings in this study improve our understanding of the molecular mechanisms of $C$. jejuni resistance to phenolic antimicrobials in association with surface polysaccharides.

\section{ACKNOWLEDGEMENTS}

This research is supported by the Natural Sciences and Engineering Research Council of Canada (NSERC). We acknowledge our gratitude to Drs Lynn McMullen and Michael Gänzle (University of Alberta) for sharing their laboratory facilities, Dr Monika Keelan (University of Alberta) for providing the C. jejuni strains PCJ497, HCJ3599 and HCJ2082, and Dr Qijing Zhang (Iowa State University) for the genomic DNA of $C$. jejuni waaF and kpsS mutants.
1 Allos, B. M. Campylobacter jejuni infections: update on emerging issues and trends. Clin. Infect. Dis. 32, 1201-1206 (2001).

2 Luangtongkum, T. et al. Antibiotic resistance in Campylobacter: emergence, transmission and persistence. Future Microbiol. 4, 189-200 (2009).

3 Payot, S. et al. Mechanisms of fluoroquinolone and macrolide resistance in Campylobacter spp. Microbes Infect. 8, 1967-1971 (2006).

4 Lin, J., Michel, L. O. \& Zhang, Q. CmeABC functions as a multidrug efflux system in Campylobacter jejuni. Antimicrob. Agents Chemother. 46, 2124-2131 (2002).

5 Gibreel, A., Skold, O. \& Taylor, D. E. Characterization of plasmid-mediated aphA-3 kanamycin resistance in Campylobacter jejuni. Microb. Drug Resist. 10, 98-105 (2004).

6 Jeon, B., Muraoka, W., Scupham, A. \& Zhang, Q. Roles of lipooligosaccharide and capsular polysaccharide in antimicrobial resistance and natural transformation of Campylobacter jejuni. J. Antimicrob. Chemother. 63, 462-468 (2009).

7 Scalbert, A., Johnson, I. T. \& Saltmarsh, M. Polyphenols: antioxidants and beyond. Am. J. Clin. Nutr. 81, 215S-217S (2005).

8 Friedman, M., Henika, P. R. \& Mandrell, R. E. Antibacterial activities of phenolic benzaldehydes and benzoic acids against Campylobacter jejuni, Escherichia coli, Listeria monocytogenes, and Salmonella enterica. J. Food Prot. 66, 1811-1821 (2003).

9 Taguri, T., Tanaka, T. \& Kouno, I. Antimicrobial activity of 10 different plant polyphenols against bacteria causing food-borne disease. Biol. Pharm. Bull. 27, 1965-1969 (2004).

10 Klancnik, A., Mozina, S. S. \& Zhang, Q. Anti-Campylobacter activities and resistance mechanisms of natural phenolic compounds in Campylobacter. PLoS One 7 e51800 (2012).

11 Hofreuter, D. et al. Unique features of a highly pathogenic Campylobacter jejuni strain. Infect. Immun. 74, 4694-4707 (2006).

12 Parkhill, J. et al. The genome sequence of the food-borne pathogen Campylobacter jejuni reveals hypervariable sequences. Nature 403, 665-668 (2000).

13 Willis, L. M. \& Whitfield, C. KpsC and KpsS are retaining 3-deoxy-D-manno-oct-2ulosonic acid (Kdo) transferases involved in synthesis of bacterial capsules. Proc. Natl Acad. Sci. USA 110, 20753-20758 (2013).

14 Oldfield, N. J., Moran, A. P., Millar, L. A., Prendergast, M. M. \& Ketley, J. M. Characterization of the Campylobacter jejuni heptosyltransferase II gene, waaF, provides genetic evidence that extracellular polysaccharide is lipid A core independent. J. Bacteriol. 184, 2100-2107 (2002).

15 Rangan, C. \& Barceloux, D. G. Food additives and sensitivities. Dis. Mon. 55, 292-311 (2009).

16 Karlyshev, A. V., Ketley, J. M. \& Wren, B. W. The Campylobacter jejuni glycome. FEMS Microbiol. Rev. 29, 377-390 (2005).

17 Nikaido, H. Molecular basis of bacterial outer membrane permeability revisited. Microbiol. Mol. Bio. Rev. 67, 593-656 (2003).

18 Vaara, M. Agents that increase the permeability of the outer membrane. Microbiol. Rev. 56, 395-411 (1992).

19 Iwata, T. et al. Effects of lipooligosaccharide inner core truncation on bile resistance and chick colonization by Campylobacter jejuni. PLOS ONE 8, e56900 (2013).

20 Yamasaki, S., Nagasawa, S., Fukushima, A., Hayashi-Nishino, M. \& Nishino, K. Cooperation of the multidrug efflux pump and lipopolysaccharides in the intrinsic antibiotic resistance of Salmonella enterica serovar Typhimurium. J. Antimicrob. Chemother. 68, 1066-1070 (2013). 